\title{
INSTANTONS AND SPECIAL GEOMETRY
}

\author{
JASON D. LOTAY AND THOMAS BRUUN MADSEN
}

\begin{abstract}
Aвstract. We survey and discuss constructions of instantons on noncompact complete manifolds of special holonomy from the viewpoint of evolution equations and give several explicit examples.
\end{abstract}

\section{To Simon Salamon on the occasion of his 60th birthday}

\section{INTRODUCTION}

Suppose we have a principal K-bundle $P \rightarrow M$ over an oriented Riemannian $n$-manifold $M$. Given a connection form $\omega \in \Omega^{1}(P ; \mathfrak{k})$ the associated curvature will be $\Omega \in \Omega^{2}(M ; \mathfrak{k})$, where $\mathfrak{k}$ is the Lie algebra of $\mathrm{K}$. When $M$ comes with a $\mathrm{G}$-structure, $\mathrm{G} \subset \mathrm{SO}(n)$, we can decompose the 2-forms as:

$$
\Lambda^{2} T^{*} M \cong \mathfrak{s o}(n) \cong \mathfrak{g} \oplus \mathfrak{g}^{\perp},
$$

where the fibres of $\mathfrak{g}$ are given by the Lie algebra of $G$. This splitting gives us a way of distinguishing connections that are particularly adapted to the geometry (cf. [26]).

Definition 1.1. A connection $\omega$ on $P$ is called a G-instanton if the 2-form part of its curvature $\Omega$ takes values in the subbundle $\mathfrak{g} \subset \Lambda^{2} T^{*} M$.

A natural setting where we have a distinguished G-structure on $M$ is when the metric on $M$ has special holonomy $G$ (and thus the G-structure is torsion-free). There are three key dimensions of manifold, and thus three holonomy groups, which will be the focus of this article: $G=S U(2)$, $\mathrm{G}_{2}$ and Spin(7). In each case we give a reformulation of the criterion of G-instanton from Definition 1.1.

For these groups $\mathrm{G}$ we unify the known constructions of $\mathrm{G}$-instantons on non-compact complete manifolds with holonomy $\mathrm{G}$ in terms of an evolution procedure. As well as bringing together examples which have occurred in the diverse literature, we analyse the limits of the instantons, including the issue of whether the instantons globally extend. We thus hope to provide insight into future constructions and classifications.

1.1. Dimension 4: $\mathrm{G}=\mathrm{SU}(2)$. On a 4-manifold $M$ we can encode the data of an $\mathrm{SU}(2)$-holonomy metric (i.e. a hyperKähler metric in 4 dimensions) in terms of a triple $\sigma=\left(\sigma_{1}, \sigma_{2}, \sigma_{3}\right)$ of 2-forms satisfying (cf. [21]):

$$
\sigma_{i} \wedge \sigma_{j}=\frac{1}{3} \delta_{i j} \sum_{k=1}^{3} \sigma_{k}^{2} \text { and } \quad d \sigma_{i}=0 .
$$

JDL was partially supported by EPSRC grant EP/K010980/1. TBM gratefully acknowledges financial support from Villum Fonden. 
The triple $\sigma$ defines a unique metric $g$ such that $\sigma$ is a triple of self-dual 2-forms and the volume form of $g$ is equal to $\frac{1}{2} \sigma_{i}^{2}$ for all $i$. The metric $g$ has holonomy contained in $\mathrm{SU}(2)$.

If we consider $\mathbb{R}^{4}$ as a representation of $\mathrm{SO}(4)$, we have

$$
\Lambda^{2} \mathbb{R}^{4} \cong \mathfrak{s o}(4)=\Lambda_{+} \oplus \Lambda_{-},
$$

where $\Lambda_{ \pm} \cong \mathfrak{s u}(2)_{ \pm} \cong \Sigma_{ \pm}^{2}$ are the \pm 1 -eigenspace of the Hodge $*$-operator. Our choice of conventions for $\mathrm{SU}(2)$-structures defined in terms of triples $\sigma$ corresponds to the choice $\mathfrak{s u}(2)=\mathfrak{s u}(2)_{-}$. We thus see that $\omega$ is an $\mathrm{SU}(2)$-instanton precisely when its curvature $\Omega$ is anti-self-dual (ASD):

$$
\Omega=-* \Omega \text {. }
$$

These connections have played an important role in the study of the topology of 4-manifolds, so one might hope that G-instantons would encode topological information in the other situations we will discuss.

1.2. Dimension 7: $\mathrm{G}=\mathrm{G}_{2}$. On a 7-manifold $M$ a $\mathrm{G}_{2}$-structure is encoded by a 3-form $\varphi$ on $M$ whose stabilizer in $\operatorname{GL}(7, \mathbb{R})$ at each point is isomorphic to $\mathrm{G}_{2}$. The form $\varphi$ defines a metric $g$ and orientation, and thus a Hodge-* operator. The torsion-free condition so that $g$ has holonomy contained in $G_{2}$ is equivalent to (by [13])

$$
d \varphi=0 \quad \text { and } \quad d * \varphi=0 .
$$

Let $V$ denote the 7-dimensional irreducible representation of $\mathrm{G}_{2}$. Then

$$
\Lambda^{2} V \cong V \oplus \mathfrak{g}_{2}
$$

There are two natural equivariant maps $\Lambda^{2} V \rightarrow \Lambda^{5} V$; one is given by the *-operator and the other comes from wedging with $\varphi$. It is easy to check that these are both isomorphisms $\Lambda^{2} V \cong \Lambda^{5} V$, and that they coincide up to a multiple of 2 on $V$ and up to a multiple of -1 on $\mathfrak{g}_{2}$. It follows that $\omega$ is a $G_{2}$-instanton precisely when its curvature satisfies

$$
\varphi \wedge \Omega=-* \Omega \text {. }
$$

Notice the similarity to Equation (1.2): this suggests that $\mathrm{G}_{2}$-instantons are in some sense natural analogues of ASD instantons in 4 dimensions.

For another useful characterization, we notice that the map obtained by wedging 2-forms with the invariant 4 -form $* \varphi$ gives an equivariant map

$$
\Lambda^{2} V \rightarrow \Lambda^{6} V \cong V \text {. }
$$

It is straightforward to check that this is an isomorphism between copies of $V$ and has kernel $\mathfrak{g}_{2} \subset \Lambda^{2} V$. So another way of phrasing that $\omega$ is a $\mathrm{G}_{2}$-instanton is that its curvature satisfies the condition

$$
* \varphi \wedge \Omega=0 .
$$

Remark 1.2. If a $\mathrm{G}_{2}$-manifold $M$ is a product $\mathbb{R}^{3} \times X$ (or $T^{3} \times X$ ) where $X$ is a hyperKähler 4-manifold with hyperKähler triple $\sigma$ then, if $\left(x^{1}, x^{2}, x^{3}\right)$ are local coordinates on $\mathbb{R}^{3}$ (or $T^{3}$ ), we have the product $\mathrm{G}_{2}$-structure

$$
\varphi=d x^{1} \wedge d x^{2} \wedge d x^{3}-d x^{1} \wedge \sigma_{1}-d x^{2} \wedge \sigma_{2}-d x^{3} \wedge \sigma_{3} .
$$


Thus, if $\omega$ is a pullback of a connection on $X$ to $M$, we see that Equation (1.4) is equivalent to Equation (1.2), since if $\Omega$ is $A S D$ on $X$ then $\Omega \wedge \sigma_{i}=0$ for all $i$. Hence, $\omega$ is a $\mathrm{G}_{2}$-instanton if and only if it is an $\mathrm{SU}(2)$-instanton.

1.3. Dimension 8: $G=\operatorname{Spin}(7)$. In a similar manner to the $G_{2}$ case just discussed, on an 8-manifold $M$ a Spin(7)-structure is equivalent to a 4form $\Phi$ on $M$ whose stabilizer in $G L(8, \mathbb{R})$ at each point is $\operatorname{Spin}(7)$. Again, $\Phi$ defines a metric $g$ and orientation, and $g$ has holonomy contained in Spin(7) if and only if (by [14])

$$
d \Phi=0 .
$$

Let $W$ denote the 8-dimensional irreducible representation of Spin(7). It is well-known that we have the orthogonal decomposition

$$
\Lambda^{2} W \cong V \oplus \mathfrak{s p i n}(7),
$$

where $V$ is the 7-dimensional irreducible complement of $\mathfrak{s p i n}(7)$ inside $\Lambda^{2} W \cong \mathfrak{s o}(8)$. Consider now the two equivariant maps $\Lambda^{2} W \rightarrow \Lambda^{6} W$ :

$$
\beta \mapsto * \beta, \quad \beta \mapsto \Phi \wedge \beta .
$$

Elementary computations show that these coincide up to a multiple of 3 on $\mathrm{V}$ and up to a multiple of -1 on $\mathfrak{s p i n}(7)$. Hence, a connection $\omega$ is a Spin(7)-instanton if and only if its curvature $\Omega$ satisfies the following:

$$
\Phi \wedge \Omega=-* \Omega \text {. }
$$

Again, notice the similarity to the ASD condition (1.2) in 4 dimensions.

Remark 1.3. If we assume that a Spin(7)-manifold $M$ is a product $\mathbb{R} \times N$ (or $\left.S^{1} \times N\right)$ where $N$ is a $\mathrm{G}_{2}$-manifold with torsion-free $\mathrm{G}_{2}$-structure $\varphi$, then if $t$ is a local coordinate on $\mathbb{R}$ (or $S^{1}$ ) we have the product $\operatorname{Spin}(7)$-structure on $M$ :

$$
\Phi=\varphi \wedge d t+*_{N} \varphi .
$$

Equations (1.4), (1.5) and (1.7) show that if $\omega$ is the pullback of a connection on $N$ to $M$ then $\omega$ is a Spin(7)-instanton if and only if it is a $\mathrm{G}_{2}$-instanton.

Remark 1.4. A related situation that shall occur is when the group $\mathrm{G}=\mathrm{SU}(3)$, as we shall see in Corollary 3.5. Here, if the SU(3)-structure on a 6-manifold is given by a 2-form $\sigma$ and 3-form $\gamma$ then $\omega$ is an $\mathrm{SU}(3)$-instanton if and only if:

$$
\Omega \wedge \gamma=0 \text { and } \Omega \wedge \sigma^{2}=0 .
$$

1.4. Construction via evolution. The construction of manifolds with special holonomy, and thus of instantons, is difficult in general, and particularly so in the compact case. This is primarily due to the analytic difficulties involved in solving systems of nonlinear PDE. However, a situation where the problem becomes tractable is where an open dense subset of $M$ is a product $I \times N$ for an interval $I \subset \mathbb{R}$. A well-known special case of this is when $M$ admits a cohomogeneity one group action.

One can identify G-structures on $I \times N$ with certain natural structures on the hypersurfaces $\{t\} \times N$, or equivalently with an $I$-family of structures on $N$. The special holonomy condition on $M$ becomes an evolution equation for the structures on $N$. Moreover, our bundle $P$ restricts to a principal K-bundle over $I \times N$, which we may always assume is the pullback of a bundle $Q \rightarrow N$. Consequently, any connection on $P$ over $I \times N$ 
can be viewed as a one-parameter family of connections on $Q$. The next elementary lemma will be instrumental in reformulating the G-instanton condition in terms of an evolution equation for the connections on $Q$.

Lemma 1.5. Let $\omega$ be a connection on a principal K-bundle over $I \times N$. Then $\omega$ can be identified with a one-parameter family $I \ni t \rightarrow A(t)$ of connections on a principal K-bundle over $N$. In particular, if $F_{A}=F_{A}(t)$ is the curvature of $A(t)$ then the curvature 2-form $\Omega$ of $\omega$ can be expressed as

$$
\Omega=d t \wedge A^{\prime}+F_{A} .
$$

Proof. A principal K-bundle $P \rightarrow I \times N$ defines a principal bundle $Q \rightarrow N$ by composing the bundle projection map with the projection $\pi: I \times N \rightarrow$ $N$; then the pullback bundle $\pi^{*} Q$ is isomorphic to $P$.

In these terms, any connection on $P$ can be written $\alpha d t+A(t)$, where we can regard $A(t)$ as a one-parameter family of connections on $Q$. The term $\alpha d t$, however, can be set to zero after performing a $t$-dependent gauge transformation $\omega \mapsto k^{-1} \omega k+k^{-1} d k$ with $k$ being the unique solution to the ODE $\partial k / \partial t=\alpha k$.

It follows that we can assume $\omega=A(t)$, and from this the expression Equation (1.9) immediately follows.

Hence, when an open dense subset of $M$ is viewed as family of hypersurfaces, the construction of instantons on this manifold with special holonomy reduces to the analysis of ODEs. Whilst this is still challenging, it could allow us to investigate key questions such as the dimension of the moduli space of instantons (including if it is non-empty) and the potential relationship between instantons and calibrated submanifolds.

Remark 1.6. The above approach can be applied to other special geometries. For instance, one can construct instantons on bundles over (open subsets of) the 8dimensional Wolf spaces $\mathbb{H P}(2), \mathrm{Gr}_{2}\left(\mathbb{C}^{4}\right)$ and $\mathrm{G}_{2} / \mathrm{SO}(4)$; these are all cohomogeneity one spaces with respect to the natural action of $\mathrm{SU}(3)$ [16].

In this case, the family of hypersurfaces consists of 7-manifolds with $\mathrm{SO}(4)$ structures in the sense of [9]. By considering suitable connections on bundles over these hypersurfaces, one obtains the type of instantons introduced in [5].

\section{THE SU(2) CASE}

2.1. SU(2)-structures. Following [3], a basic construction of metrics with $\mathrm{SU}(2)$ holonomy begins with an oriented 3-manifold $N$ equipped with a one-parameter family of oriented coframes

$$
I \ni t \rightarrow e(t)=\left(e^{1}(t), e^{2}(t), e^{3}(t)\right),
$$

so that $e^{1}(t) \wedge e^{2}(t) \wedge e^{3}(t)>0$. These coframes are declared to be orthonormal so that we have a family of induced metrics on $N$ given by $g(t)=e^{1}(t)^{2}+e^{2}(t)^{2}+e^{3}(t)^{2}$. From this family of coframes, we can construct a triple $\sigma$ of 2 -forms on the product $M=I \times N$ :

$$
\sigma_{1}=d t \wedge e^{1}+e^{2} \wedge e^{3}, \sigma_{2}=d t \wedge e^{2}+e^{3} \wedge e^{1}, \sigma_{3}=d t \wedge e^{3}+e^{1} \wedge e^{2} .
$$


These forms are self-dual with respect to $d t^{2}+g(t)$ and satisfy the first equations in (1.1). In terms of data on $N$ the condition $d \sigma=0$ amounts to

$$
d *_{t} e(t)=0,
$$

where $*_{t}$ is the Hodge star given by $g(t)$ and the orientation $e^{1}(t) \wedge e^{2}(t) \wedge$ $e^{3}(t)$, for each $t$ together with the equations:

$$
\left(*_{t} e\right)^{\prime}=\text { de. }
$$

As the condition $d * e=0$ is preserved by Equation (2.2), we can in a sense regard Equation (2.2) as a way of evolving an initial co-closed coframe on $N$. This is sometimes called an "SU(2)-flow" though it is not in any sense a parabolic equation, and so does not satisfy the usual analytic properties one would expect of a geometric flow (cf. [3]).

In addition to the flat metric on $\mathbb{R}^{4}$ there are basically three interesting metrics arising directly from this construction (see, for instance, [17, Proposition 2.7]): the Eguchi-Hanson metric, the Taub-NUT metric and the Atiyah-Hitchin metric. These metrics are complete, have full holonomy $\mathrm{SU}(2)$, and are examples of gravitational instantons.

2.2. SU(2)-instantons. If $M=I \times N$ and the bundle $P$ is the pullback of $Q \rightarrow N$, then we can express the $\mathrm{SU}(2)$-instanton condition using Lemma 1.5 and Equation (1.2) as follows.

Proposition 2.1. A connection $\omega$ on $P$ over $I \times N$ is an $\mathrm{SU}(2)$-instanton if and only if the one-parameter family $A(t)$ of connections on $Q \rightarrow N$ satisfies:

$$
A^{\prime}(t)=-*_{t} F_{A}(t) .
$$

As Equation (2.3) is in Cauchy form, we immediately deduce:

Corollary 2.2. Given real-analytic initial data, the SU(2)-instanton evolution equation (2.3) admits a unique solution over an open subset of $I \times N$.

Corollary 2.3. If $\omega$ is asymptotic to a connection on $Q \rightarrow N$ at an endpoint of $I$, then the limiting connection is flat.

Proof. From the form of $\Omega$, we see that it can approach the curvature of a connection on $N$ at an endpoint of $I$ only if $A^{\prime} \rightarrow 0$, and in that case Equation (2.3) implies that $F_{A} \rightarrow 0$.

2.3. Flat $\mathbb{R}^{4}$. It is easy to see that if $\eta=\left(\eta^{1}, \eta^{2}, \eta^{3}\right)$ is the standard leftinvariant coframe on $S^{3}=\mathrm{SU}(2)$ with $d \eta^{1}=2 \eta^{2} \wedge \eta^{3}$ etc. and $\eta^{1} \wedge \eta^{2} \wedge$ $\eta^{3}>0$, we have a solution $e(t)=t \eta$ to Equation (2.2) with corresponding SU(2) triple $\sigma$ given by $\sigma_{1}=t d t \wedge \eta^{1}+t^{2} \eta^{2} \wedge \eta^{3}$ etc. If we take $P=$ $\mathrm{SU}(2) \times \mathbb{R}^{4}$, we can view the connections $A(t)$ on $Q=\mathrm{SU}(2) \times S^{3}$ as triples of 1 -forms on $Q$.

The simplest case is when $A(t)=a(t) e(t)=a t \eta$, so $F_{A}(t)=a t(1+a t) d \eta$ and thus Equation (2.3) is equivalent to

$$
(a t)^{\prime}=-\frac{2 a t(1+a t)}{t}
$$

which has solutions

$$
a(t)=-\frac{k}{t\left(t^{2}+k\right)}
$$


for $k \in \mathbb{R}$. For non-trivial solutions defined on all of $\mathbb{R}^{4}$ we take $k>0$. Then the corresponding SU(2)-instantons have curvature

$$
\Omega=\frac{2 k}{\left(t^{2}+k\right)^{2}}\left(d t \wedge e^{1}-e^{23}, d t \wedge e^{2}-e^{31}, d t \wedge e^{3}-e^{12}\right) .
$$

Taking $k=1$ gives the basic instanton over $\mathbb{R}^{4}$. Notice that, indeed, the connection is asymptotic at infinity to a flat connection over $S^{3}$ as predicted by Corollary 2.3.

2.4. Eguchi-Hanson. In Section 2.3 we have somewhat implicitly used the fact that $\mathbb{R}^{4}$ is the completion of $\mathbb{R}_{+} \times S^{3}$. In this case, it is evident that the flat hyperKähler structure and (basic) instanton are defined on the whole of $\mathbb{R}^{4}$ and $P$, respectively. In less elementary cases, more care needs to be taken if we want to make sure our structures are defined everywhere.

In order to illustrate this, let us look at the Eguchi-Hanson metric as derived in [8]. To this end, we consider a basis $\left\{\eta^{j}\right\}$ for $\mathfrak{s o}(3)$ with $d \eta^{1}=\eta^{23}$ etc. and $\eta^{1} \wedge \eta^{2} \wedge \eta^{3}>0$. We then make the ansatz $d t=f^{-1}(r) d r$, $e^{1}(r)=r f^{-1}(r) \eta^{1}, e^{2}(r)=f(r) \eta^{2}$ and $e^{3}(r)=f(r) \eta^{3}$. Given this, Equation (2.1) is equivalent to the ODE

$$
\frac{\partial\left(f^{2}\right)}{\partial r}=r f^{-2}
$$

which (up to sign) has the solution $f(r)=\left(k+r^{2}\right)^{1 / 4}$, where $k \in \mathbb{R}$. To get the Eguchi-Hanson metric on $T^{*} S^{2}$, we should take $k>0$. Taking $k=0$ gives the flat metric on $\mathbb{R}^{4} /\{ \pm 1\}$, and $k<0$ leads to an incomplete metric.

In this Eguchi-Hanson space the principal orbits are $\mathrm{SO}(3)$ and the singular orbit is $S^{2}=\mathrm{SO}(3) / \mathrm{SO}(2)$. To understand the behaviour of the metric near the singular orbit, we consider the vector bundle

$$
\mathbb{V}=\mathrm{SO}(3) \times{ }_{\mathrm{SO}(2)} V ;
$$

here the fibres $V$ correspond to the standard representation of $\mathrm{SO}(2)$. We shall write $T=\left\langle\eta^{2}, \eta^{3}\right\rangle$ for the $\operatorname{Ad}_{\mathrm{SO}(2)}$-invariant complement $\mathfrak{s o}(2)^{\perp} \subset$ $\mathfrak{s o}(3)$. The $\mathrm{SO}(3)$-invariant forms on $\mathbb{V}$ are the elements of $\left(\Lambda^{*} T\right)^{\mathrm{SO}(2)}$ together with "words" whose syllables come about by contracting "letters" (using the inner product an volume form on $\mathbb{R}^{2}$ )

$$
\boldsymbol{a}=\left(\begin{array}{l}
a_{1} \\
a_{2}
\end{array}\right), \quad \boldsymbol{b}=\left(\begin{array}{l}
b_{1} \\
b_{2}
\end{array}\right)=\left(\begin{array}{l}
d a_{1}+a_{2} \eta^{1} \\
d a_{2}-a_{1} \eta^{1}
\end{array}\right), \quad \boldsymbol{c}=\left(\begin{array}{l}
c_{1} \\
c_{2}
\end{array}\right)=\left(\begin{array}{l}
\eta^{2} \\
\eta^{3}
\end{array}\right),
$$

where $a_{1}, a_{2}$ denote fibre coordinates, and $\boldsymbol{b}$ is the covariant derivative of a. For example, the following four 2 -forms are $\mathrm{SO}(3)$-invariant:

$$
\begin{gathered}
\Sigma(\boldsymbol{b}, \boldsymbol{b})=-b_{1} b_{2}, \quad \Sigma(\boldsymbol{c}, \boldsymbol{c})=c_{1} c_{2}, \\
\boldsymbol{b} \boldsymbol{c}=b_{1} c_{1}+b_{2} c_{2}, \quad \Sigma(\boldsymbol{b}, \boldsymbol{c})=b_{1} c_{2}-b_{2} c_{1} .
\end{gathered}
$$

The map $\Psi: \mathrm{SO}(3) \times \mathbb{R} \rightarrow \mathrm{SO}(3) \times V$ given by $\Psi(g, r)=(g,(r, 0))$ induces a map $\mathrm{SO}(3) \times \mathbb{R} \rightarrow \mathbb{V}$ that we can use to express the 2-forms $\sigma_{j}$ of Equation (2.1) in terms of words from the "dictionary". By noting that $\Psi^{*}\left(\eta^{j}\right)=\eta^{j}$ for $j=2,3$ and $\Psi^{*}\left(b_{1}\right)=d r, \Psi^{*}\left(b_{2}\right)=-r \eta^{1}$, we get that

$$
\sigma_{1}=f^{-2} \Sigma(\boldsymbol{b}, \boldsymbol{b})+f^{2} \Sigma(\boldsymbol{c}, \boldsymbol{c}), \quad \sigma_{2}=\Sigma(\boldsymbol{b}, \boldsymbol{c}), \quad \sigma_{3}=\boldsymbol{b} \boldsymbol{c} .
$$


For $k>0$, the fact that these forms extend to the zero section follows immediately by observing that the coefficient functions are smooth even functions of the distance from the zero section of $\mathbb{V}$.

More generally, [7, Thm. 4.1] gives that any $\mathrm{SO}(3)$-invariant 2-form on $\mathbb{V} \backslash S^{2}$ can be expressed in terms of

$$
\begin{aligned}
k_{1} \boldsymbol{b} \boldsymbol{c}+ & k_{2} \Sigma(\boldsymbol{b}, \boldsymbol{b})+k_{3} \Sigma(\boldsymbol{b}, \boldsymbol{c})+k_{4} \Sigma(\boldsymbol{c}, \boldsymbol{c}) \\
+ & k_{5} \boldsymbol{a} \boldsymbol{b} \boldsymbol{a} \boldsymbol{c}+k_{6} \Sigma(\boldsymbol{a}, \boldsymbol{b}) \boldsymbol{a c} .
\end{aligned}
$$

Such a 2-form extends smoothly to the zero section if and only if the coefficient functions $k_{i}$ are smooth even functions of the radial coordinate $r=\sqrt{\boldsymbol{a a}}$; this follows by applying the arguments of [11, Lemma 1.1] and by observing that the basis of Equation (2.5) is adapted to the filtration of $\mathrm{SO}(2)$-equivariant homogeneous polynomials $V \supset S^{2} \rightarrow \Lambda^{2}(T \oplus V)$.

Let us consider $\mathrm{SO}(3)$-invariant instantons on the natural circle bundle over the Eguchi-Hanson space whose total space is $\mathrm{SO}(3) \times V$. Away from the zero section we can describe our connection in terms of a potential given by

It follows that

$$
A(r)=p(r) e^{1}
$$

$$
A^{\prime}=f\left(\frac{\partial p}{\partial r}+\frac{f}{r} \frac{\partial\left(r f^{-1}\right)}{\partial r} p\right) e^{1}
$$

and that the curvature 2-form $F_{A}$, on each hypersurface, is given by $F_{A}=$ $r f^{-3} p e^{23}$. In particular, we have $*_{t} F_{A}=r f^{-3} p e^{1}$.

Altogether Equation (2.3) therefore amounts to the following ODE

$$
\frac{\partial p}{\partial r}=-\left(\frac{r}{f^{4}}+\frac{f}{r} \frac{\partial\left(r f^{-1}\right)}{\partial r}\right) p .
$$

If, for concreteness, we take $f(r)=\left(1+r^{2}\right)^{1 / 4}$, then this equation has the solution

$$
p(r)=\frac{c}{r\left(1+r^{2}\right)^{1 / 4}}
$$

for $c \in \mathbb{R}$. Computing the associated curvature 2-form we find

$$
\Omega=-\frac{c}{1+r^{2}}\left(d t \wedge e^{1}-e^{23}\right)=-\frac{c}{1+\boldsymbol{a} \boldsymbol{a}}\left(f^{-2} \Sigma(\boldsymbol{b}, \boldsymbol{b})-f^{2} \Sigma(\boldsymbol{c}, \boldsymbol{c})\right),
$$

showing that our solution is asymptotically flat.

By generalising our computations slightly, we find that the above instanton is unique in the following sense:

Proposition 2.4. There is a unique $\mathrm{SO}(3)$-invariant $\mathrm{SU}(2)$-instanton on the natural circle bundle over the Eguchi-Hanson space whose curvature at the zero section restricts to that of the canonical connection $\mathrm{SO}(3) \rightarrow S^{2}$.

As the distance from the zero section increases, this connection approaches a flat connection on a circle bundle over $\mathrm{SO}(3)$.

Remark 2.5. In light of Proposition 2.4, it is tempting to think of the instanton evolution equations as a singular initial value problem, prescribing initial data at the singular orbit as in [11]. This approach, however, still requires knowledge about the explicit solution: when we express our connection (or curvature) in terms of a basis adapted to the filtration of equivariant homogeneous polynomials, 
we still need to verify that the coefficient functions are smooth even functions of the distance from the zero section.

2.5. Taub-NUT. So far we have only considered left-invariant coframes on $\mathrm{SU}(2)$. If we instead view a coframe as a 1-form taking values in the imaginary quaternions $e=i e^{1}+j e^{2}+k e^{3}$ and suppose that $e=q \epsilon q^{-1}$ where $\epsilon$ is left-invariant, then if $\eta=i \eta^{1}+j \eta^{2}+k \eta^{3}$ is the standard leftinvariant coframe on $\mathrm{SU}(2)$, as in Section 2.3, we see that

$$
\begin{gathered}
q^{-1}(d e) q=i\left(d \epsilon^{1}-2 \epsilon^{2} \wedge \eta^{3}+2 \epsilon^{3} \wedge \eta^{2}\right)+j\left(d \epsilon^{2}-2 \epsilon^{3} \wedge \eta^{1}+2 \epsilon^{1} \wedge \eta^{3}\right) \\
+k\left(d \epsilon^{3}-2 \epsilon^{1} \wedge \eta^{2}+2 \epsilon^{2} \wedge \eta^{1}\right) .
\end{gathered}
$$

As is well-known (see e.g. [1]), if we take $\epsilon=i f_{1} \eta^{1}+j f_{2} \eta^{2}+k f_{3} \eta^{3}$ then Equation (2.2) is equivalent to $\left(f_{2} f_{3}\right)^{\prime}=2\left(f_{1}-f_{2}-f_{3}\right)$ etc. Making the ansatz $d t=-\frac{1}{2}(r+m) f^{-1}(r) d r, f_{1}=2 m(r+m)^{-1} f(r)$ and $f_{2}=f_{3}=f(r)$ for a constant $m \geqslant 0$ and function $f$ quickly yields the ODE

$$
\frac{\partial\left(f^{2}\right)}{\partial r}=2 r
$$

The solution (up to sign) defined for $r \geqslant m$ is $f(r)=\left(r^{2}-m^{2}\right)^{\frac{1}{2}}$, which gives the so-called Taub-NUT metric (with mass $m$ ) defined on $\mathbb{R}^{4}$. The unit coframe for each $r$ is given by $q \epsilon q^{-1}$ where

$$
\epsilon=i \epsilon^{1}+j \epsilon^{2}+k \epsilon^{3}=2 m\left(\frac{r-m}{r+m}\right)^{\frac{1}{2}} i \eta^{1}+\left(r^{2}-m^{2}\right)^{\frac{1}{2}}\left(j \eta^{2}+k \eta^{3}\right) .
$$

We can now study instantons on say the trivial SU(2)-bundle over TaubNUT. The natural family of connections on $S^{3}$ to consider is

$$
A(r)=q\left(a(r) i \eta^{1}+b(r) j \eta^{2}+b(r) k \eta^{3}\right) q^{-1} .
$$

The curvature is then given by

$$
q^{-1} F_{A} q=2 i\left(a-2 b+b^{2}\right) \eta^{2} \wedge \eta^{3}+2 j a(b-1) \eta^{3} \wedge \eta^{1}+2 k a(b-1) \eta^{1} \wedge \eta^{2} .
$$

We readily find that the instanton evolution (2.3) is equivalent to

$$
\frac{\partial a}{\partial r}=\frac{2 m\left(a-2 b+b^{2}\right)}{r^{2}-m^{2}} \quad \text { and } \quad \frac{\partial b}{\partial r}=\frac{a(r+m)(b-1)}{2 m(r-m)} .
$$

There is an obvious solution with $b=1$ and, for a constant $c$,

$$
a=1+c \frac{r-m}{r+m} \text {. }
$$

Using the notation of Equation (2.6) we obtain the following.

Proposition 2.6. The connection on the Taub-NUT space with mass m given by

$$
\omega=q\left(\frac{1}{2 m}\left(\frac{r+m}{r-m}\right)^{\frac{1}{2}}\left(1+c \frac{r-m}{r+m}\right) i \epsilon^{1}+\frac{1}{\left(r^{2}-m^{2}\right)^{\frac{1}{2}}}\left(j \epsilon^{2}+k \epsilon^{3}\right)\right) q^{-1}
$$


for a constant $c$ is an $\mathrm{SU}(2)$-instanton. The connection blows up at the "nut" $r=m$ and the curvature is

$$
\begin{aligned}
\Omega & =\frac{c}{(r+m)^{2}} q i q^{-1}\left(\left(\frac{r+m}{r-m}\right)^{\frac{1}{2}} d r \wedge \epsilon^{1}+2 \epsilon^{2} \wedge \epsilon^{3}\right) \\
& =-\frac{2 c}{(r+m)^{2}} q i q^{-1}\left(d t \wedge \epsilon^{1}-\epsilon^{2} \wedge \epsilon^{3}\right),
\end{aligned}
$$

which shows that $\omega$ is asymptotic to the flat connection on $S^{3}$ as $r \rightarrow \infty$.

Notice that we just get the flat connection on Taub-NUT when $c=0$.

Remark 2.7. We can perform the same study for the Atiyah-Hitchin metric [1], where $f_{1}, f_{2}, f_{3}$ are distinct, obtaining ODEs describing $\mathrm{SU}(2)$-instantons. The analysis of these ODEs is more involved, so we do not pursue this here.

\section{THE $\mathrm{G}_{2}$ CASE}

3.1. $\mathrm{G}_{2}$-structures. In this setting we consider an oriented 6-manifold $N$ equipped with a one-parameter family of $\mathrm{SU}(3)$-structures

$$
I \ni t \mapsto(\sigma(t), \gamma(t)) ;
$$

for each $t$, the pair $(\sigma(t), \gamma(t)) \in \Omega^{2}(N) \times \Omega^{3}(N)$ determines a reduction of the principal frame bundle to an $\mathrm{SU}(3)$-subbundle. Recall that for each $t$ the $\mathrm{SU}(3)$-structure determines $\hat{\gamma}(t) \in \Omega^{3}(N)$ such that $\gamma+i \hat{\gamma}$ is a nowhere vanishing $(3,0)$-form on $N$. From this family, we can build a $\mathrm{G}_{2}$-structure on the product $M=I \times N$ by setting

$$
\left\{\begin{array}{l}
\varphi=\sigma \wedge d t+\gamma \\
* \varphi=\hat{\gamma} \wedge d t+\frac{1}{2} \sigma^{2} .
\end{array}\right.
$$

The torsion-free condition Equation (1.3) then amounts to requiring that each SU(3)-structure $(\sigma(t), \gamma(t))$ is half-flat, meaning

$$
d \sigma^{2}=0=d \gamma,
$$

and that the family satisfies "Hitchin's flow equations" [20]:

$$
\frac{\partial \gamma}{\partial t}=d \sigma, \quad \frac{\partial \sigma^{2}}{\partial t}=-2 d \hat{\gamma}
$$

As the condition Equation (3.2) of being half-flat is preserved by Equation (3.3), we can regard these equations as a way of evolving an initial half-flat SU(3)-structure on $N$ to construct a metric with holonomy contained in $\mathrm{G}_{2}$. Again, the "flow" terminology is somewhat specious given the system's lack of parabolicity and the results in [3].

3.2. $\mathrm{G}_{2}$-instantons. Given a $\mathrm{G}_{2}$-manifold $M=I \times N$ as in Equation (3.1), we can rewrite the $G_{2}$-instanton condition on a connection $\omega$ on the pullback of a bundle $Q$ on $N$ as follows:

Lemma 3.1. In terms of $t$-dependent data on $N$, the $\mathrm{G}_{2}$-instanton equation (1.4) is equivalent to the condition

$$
\left\{\begin{array}{l}
A^{\prime}=*_{t}\left(F_{A} \wedge \gamma\right) \\
\sigma \wedge F_{A}+*_{t} F_{A}=\gamma \wedge *_{t}\left(F_{A} \wedge \gamma\right) .
\end{array}\right.
$$


The alternative condition (1.5) can be rephrased as

$$
\left\{\begin{array}{l}
A^{\prime} \wedge \sigma^{2}=2 F_{A} \wedge \hat{\gamma} \\
F_{A} \wedge \sigma^{2}=0 .
\end{array}\right.
$$

Proof. Using Lemma 1.5, the left hand side of Equation (1.4) can be written:

$$
(\sigma \wedge d t+\gamma) \wedge\left(d t \wedge A^{\prime}+F_{A}\right)=d t \wedge\left(\sigma \wedge F_{A}-\gamma \wedge A^{\prime}\right)+F_{A} \wedge \gamma
$$

The right hand side of Equation (1.4) reads

$$
-* \Omega=-*\left(d t \wedge A^{\prime}\right)-*\left(F_{A}\right) .
$$

This tells us that

$$
-*_{t} A^{\prime}=F_{A} \wedge \gamma \quad \text { and } \quad-*_{t}\left(F_{A}\right)=\sigma \wedge F_{A}-\gamma \wedge A^{\prime} .
$$

Clearly, these two expressions are equivalent to Equation (3.4). Similarly,

$$
\begin{aligned}
* \varphi \wedge \Omega & =\left(\hat{\gamma} \wedge d t+\frac{1}{2} \sigma^{2}\right) \wedge\left(d t \wedge A^{\prime}+F_{A}\right) \\
& =d t \wedge\left(\frac{1}{2} A^{\prime} \wedge \sigma^{2}-F_{A} \wedge \hat{\gamma}\right)+F_{A} \wedge \frac{1}{2} \sigma^{2},
\end{aligned}
$$

from which Equation (3.5) follows.

It is then a question of straightforward computations to obtain:

Proposition 3.2. The evolution equations for $\mathrm{G}_{2}$-instantons may be phrased as:

$$
\left\{\begin{array}{l}
A^{\prime}=*_{t}\left(F_{A} \wedge \gamma\right) \\
F_{A}\left(t_{0}\right) \wedge \sigma^{2}\left(t_{0}\right)=0,
\end{array}\right.
$$

for some initial $t_{0} \in I$.

Proof. Let $W$ be the 6-dimensional irreducible representation of SU(3). Elementary computations show that the equivariant maps $\Lambda^{2} W \rightarrow \Lambda^{5} W$ given by

$$
\beta \mapsto \sigma^{2} \wedge *(\beta \wedge \gamma) \text { and } \beta \mapsto 2 \beta \wedge \hat{\gamma}
$$

coincide. It therefore follows that the evolution of $A$ is completely determined by the equation for $A^{\prime}$ in Equation (3.4).

Next, we show that if a 2 -form $\beta$ has $\beta \wedge \sigma^{2}=0$ then it automatically satisfies the constraint of Equation (3.4), that is,

$$
\sigma \wedge \beta+* \beta=\gamma \wedge *(\beta \wedge \gamma)
$$

This is because the two equivariant maps $\Lambda^{2} W \rightarrow \Lambda^{4} W$ given by

$$
\beta \mapsto \sigma \wedge \beta+* \beta \text { and } \beta \mapsto \gamma \wedge *(\beta \wedge \gamma)
$$

agree on the two irreducible submodules orthogonal to $\langle\sigma\rangle$ in $\Lambda^{2} W$.

In order to prove the proposition, we need to show that the 6-form $F_{A} \wedge \sigma^{2}$ is preserved as $A$ evolves. This assertion follows by

$$
\begin{aligned}
\left(F_{A} \wedge \sigma^{2}\right)^{\prime} & =F_{A}^{\prime} \wedge \sigma^{2}+F_{A} \wedge\left(\sigma^{2}\right)^{\prime} \\
& =\left(d A^{\prime}+\left[A, A^{\prime}\right]\right) \wedge \sigma^{2}+F_{A} \wedge(-2 d \hat{\gamma}) \\
& =2 d F_{A} \wedge \hat{\gamma}+2 F_{A} \wedge d \hat{\gamma}+2\left[A, F_{A}\right] \wedge \hat{\gamma}-2 F_{A} \wedge d \hat{\gamma} \\
& =2\left(d F_{A}+\left[A, F_{A}\right]\right) \wedge \hat{\gamma}=2 D F_{A} \wedge \hat{\gamma}=0,
\end{aligned}
$$

where the last equality follows from the Bianchi identity. 
Remark 3.3. The condition $F_{A}\left(t_{0}\right) \wedge \sigma^{2}\left(t_{0}\right)=0$ in Proposition 3.2 is not very restrictive: it simply means that the 2-form part of $F_{A}\left(t_{0}\right)$ is not allowed to have a component (pointwise) proportional to $\sigma\left(t_{0}\right)$.

Note that the evolution equation for $A$ is in Cauchy form, which means that we immediately have:

Corollary 3.4. Given real analytical initial data $A$ on $N$ satisfying the condition $F_{A} \wedge \sigma^{2}=0$, the $\mathrm{G}_{2}$-instanton evolution equations (3.6) have a unique solution over an open set in $I \times N$.

From the form of Equation (3.6), we have the following:

Corollary 3.5. If a $\mathrm{G}_{2}$-instanton $\omega$ is asymptotic to a connection on $Q \rightarrow N$ at an endpoint of $I$, then the limiting connection is an SU(3)-instanton.

Proof. As we must have $A^{\prime} \rightarrow 0$, we have

$$
F_{A} \wedge \gamma \rightarrow 0 \text { and } F_{A} \wedge \sigma^{2}=0,
$$

which means that $A$ tends to a connection whose curvature has values in $\mathfrak{s u}(3) \subset \mathfrak{s o}(6) \cong \Lambda^{2}$, which is an $\mathrm{SU}(3)$-instanton as in Equation (1.8).

3.3. Flat $\mathbb{R}^{7}$. To construct the flat metric on $\mathbb{R}^{7}$, which corresponds to a trivial torsion-free $\mathrm{G}_{2}$-structure, we need a half-flat $\mathrm{SU}(3)$-structure $(\sigma, \gamma)$ on $S^{6}$ : this is provided by the standard nearly Kähler structure on $S^{6}$ which satisfies

$$
d \sigma=3 \gamma \quad \text { and } \quad d \hat{\gamma}=-2 \sigma^{2}
$$

The evolution equations (3.3) starting at a nearly Kähler structure always lead to the solution:

$$
\varphi=t^{2} \sigma \wedge d t+t^{3} \gamma \quad \text { and } \quad * \varphi=t^{3} \hat{\gamma} \wedge d t+\frac{1}{2} t^{4} \sigma^{2}
$$

for $t>0$, yielding the cone metric over the nearly Kähler manifold. Using this description of $\mathbb{R}^{7}$ together with an appropriate ansatz for $A$, one can use the evolution equation (3.6) to give a higher dimensional generalisation of the basic instanton on $\mathbb{R}^{4}$. Indeed, we can reconstruct the instanton on the trivial bundle $G_{2} \times \mathbb{R}^{7}$ described in $[19,22]$.

There are other ways to think of $\mathbb{R}^{7}$ as a family of hypersurfaces. For example, we could think of $\mathbb{R}^{7}=\mathbb{R}^{3} \times \mathbb{R}^{4}$ and take hypersurfaces $\mathbb{R}^{3} \times S^{3}$. If $\left(x^{1}, x^{2}, x^{3}\right) \in \mathbb{R}^{3}$ and $\eta^{1}, \eta^{2}, \eta^{3}$ is our standard left-invariant coframe on $S^{3}$, our ansatz for the evolving $\mathrm{SU}(3)$-structures on $\mathbb{R}^{3} \times S^{3}$ would be

$$
\begin{aligned}
& \sigma=f(t)\left(d x^{1} \wedge \eta^{1}+d x^{2} \wedge \eta^{2}+d x^{3} \wedge \eta^{3}\right), \\
& \gamma=d x^{123}-f(t)^{2}\left(d x^{1} \wedge \eta^{2} \wedge \eta^{3}+d x^{2} \wedge \eta^{3} \wedge \eta^{1}+d x^{3} \wedge \eta^{1} \wedge \eta^{2}\right) .
\end{aligned}
$$

The evolution equations (3.3) quickly yield a solution $f(t)=t$ for $\varphi=$ $\sigma \wedge d t+\gamma$ to be torsion-free. We see that this is equivalent to allowing the coframe on $S^{3}$ to evolve as $e(t)=t \eta$ just as in the flat $\mathbb{R}^{4}$ case, as expected. If we take evolving connections $A(t)=a(t) t \eta$ on $\mathbb{R}^{3} \times S^{3}$, the $\mathrm{G}_{2}$-instanton evolution equations give Equation (2.4) so that $\omega$ is the pullback of the basic instanton on $\mathbb{R}^{4}$, which is clear in light of Remark 1.2.

Alternatively, we could view $\mathbb{R}^{7}$ in terms of hypersurfaces $S^{2} \times \mathbb{R}^{4} \subseteq$ $\mathbb{R}^{3} \times \mathbb{R}^{4}$, and perform the same analysis to get the flat metric by evolving 
the volume form on $S^{2}$ by the obvious scaling. In this case, the pullback of an evolving connection on $S^{2}$ to $S^{2} \times \mathbb{R}^{4}$ will define a $G_{2}$-instanton if and only if the corresponding connection on $\mathbb{R}^{3}$ is flat. This situation is equivalent to considering $\mathbb{R}^{7}=\Lambda_{+}^{2} \mathbb{R}^{4}$ and having the sphere subbundles as hypersurfaces. We shall see a related construction in the next section which yields non-trivial results.

3.4. Self-dual 2-forms over $S^{4}$. Following [4,7], we first describe a space that in a sense is related to that of Eguchi-Hanson, described in Section 2.1. In this case, we are considering a manifold with a cohomogeneity one action of $\mathrm{SO}(5)$. The principal stabiliser is $\mathrm{U}(2) \subset \mathrm{SO}(4)$, and the singular stabiliser is the whole subgroup $\mathrm{SO}(4)$.

We shall need a suitable local coframe on $\mathbb{C P}(3)=\mathrm{SO}(5) / \mathrm{U}(2)$. Let us write $\mathfrak{s o}(5)=\left\langle v^{1}, v^{2}, v^{3}, v^{4}\right\rangle \oplus\left\langle\eta^{1}, \eta^{2}, \eta^{2}\right\rangle \oplus\left\langle\gamma^{1}, \gamma^{2}, \gamma^{3}\right\rangle$ in terms of the explicit identification:

$$
\left(\begin{array}{ccccc}
0 & -\frac{1}{2}\left(\eta^{1}+\gamma^{1}\right) & -\frac{1}{2}\left(\eta^{2}+\gamma^{2}\right) & -\frac{1}{2}\left(\eta^{3}+\gamma^{3}\right) & -v^{1} \\
\frac{1}{2}\left(\eta^{1}+\gamma^{1}\right) & 0 & -\frac{1}{2}\left(\eta^{3}-\gamma^{3}\right) & \frac{1}{2}\left(\eta^{2}-\gamma^{2}\right) & -v^{2} \\
\frac{1}{2}\left(\eta^{2}+\gamma^{2}\right) & \frac{1}{2}\left(\eta^{3}-\gamma^{3}\right) & 0 & -\frac{1}{2}\left(\eta^{1}-\gamma^{1}\right) & -v^{3} \\
\frac{1}{2}\left(\eta^{3}+\gamma^{3}\right) & -\frac{1}{2}\left(\eta^{2}-\gamma^{2}\right) & \frac{1}{2}\left(\eta^{1}-\gamma^{1}\right) & 0 & -v^{4} \\
v^{1} & v^{2} & v^{3} & v^{4} & 0
\end{array}\right)
$$

Choosing $u(2)=\left\langle\eta^{1}, \gamma^{1}, \gamma^{2}, \gamma^{3}\right\rangle$, we have $d$ acting as:

$$
\begin{gathered}
d \eta^{1}=-\eta^{23}-\left(v^{12}+v^{34}\right), \\
d \eta^{2}=-\eta^{31}-\left(v^{13}+v^{42}\right), d \eta^{3}=-\eta^{12}-\left(v^{14}+v^{23}\right), \\
d\left(v^{12}+v^{34}\right)=-\left(v^{14}+v^{23}\right) \eta^{2}+\left(v^{13}+v^{42}\right) \eta^{3}, \\
d\left(v^{13}+v^{42}\right)=\left(v^{14}+v^{23}\right) \eta^{1}-\left(v^{12}+v^{34}\right) \eta^{3}, \\
d\left(v^{14}+v^{23}\right)=-\left(v^{13}+v^{42}\right) \eta^{1}+\left(v^{12}+v^{34}\right) \eta^{2} .
\end{gathered}
$$

We now look for an ansatz with $d t=f^{-1}(r) d r, e^{1}(r)=r f^{-1} \eta^{2}, e^{2}(r)=$ $r f^{-1} \eta^{3}$ and $e^{3}(r)=f v^{1}, e^{4}(r)=-f v^{2}, e^{5}(r)=f v^{4}, e^{6}(r)=f v^{3}$ so that

$$
\begin{gathered}
\sigma(r)=\left(r f^{-1}\right)^{2} \eta^{23}-f^{2}\left(v^{12}+v^{34}\right), \\
\gamma(r)=r f\left(\left(v^{14}+v^{23}\right) \eta^{2}-\left(v^{13}+v^{42}\right) \eta^{3}\right), \\
\hat{\gamma}(r)=r f\left(\left(v^{13}+v^{42}\right) \eta^{2}+\left(v^{14}+v^{23}\right) \eta^{3}\right) .
\end{gathered}
$$

In this case, Equation (3.3) reduces to the following ODE

$$
\frac{\partial f}{\partial r}=r f^{-3}
$$

which has the solution $f(r)=2^{1 / 4}\left(k+r^{2}\right)^{1 / 4}$, for some constant $k \in \mathbb{R}$. To get the Bryant-Salamon metric on $\Lambda_{+}^{2} S^{4}$ we must take $k>0$.

As for the Eguchi-Hanson case, we next consider a tubular neighbourhood of the singular orbit. This is modelled on the vector bundle

$$
\mathbb{V}=\mathrm{SO}(5) \times{ }_{\mathrm{SO}(4)} V
$$

with fibres $V \cong\left\langle v^{12}+v^{34}, v^{13}+v^{42}, v^{14}+v^{23}\right\rangle=\Lambda_{+}^{2}(T), T \cong \mathfrak{s o}(5) / \mathfrak{s o}(4)$. 
In this case, we can form the words

$$
\boldsymbol{a}=\left(\begin{array}{l}
a_{1} \\
a_{2} \\
a_{3}
\end{array}\right), \quad \boldsymbol{b}=\left(\begin{array}{l}
d a_{1}+a_{3} \eta^{2}-a_{2} \eta^{3} \\
d a_{2}-a_{3} \eta^{1}+a_{1} \eta^{3} \\
d a_{3}+a_{2} \eta^{1}-a_{1} \eta^{2}
\end{array}\right), \quad \boldsymbol{c}=\left(\begin{array}{c}
v^{12}+v^{34} \\
v^{13}+v^{42} \\
v^{14}+v^{23}
\end{array}\right) .
$$

The map $\Psi: \mathrm{SO}(5) \times \mathbb{R} \rightarrow \mathrm{SO}(5) \times V$, given by $\Psi(g, r)=(g,(r, 0,0))$, induces a map $\mathbb{C P}(3) \times \mathbb{R} \rightarrow \mathbb{V}$ that we can use to express the 3-form $\varphi$ and 4 -form $* \varphi$ in terms of words from the dictionary. For this, we note that $\Psi^{*}\left(b_{1}\right)=d r, \Psi^{*}\left(b_{2}\right)=r \eta^{3}, \Psi^{*}\left(b_{3}\right)=-r \eta^{2}$. It then follows that, up to suitable rescaling of invariant forms (similar to the Eguchi-Hanson case, these are obtained via contractions coming from the inner product and volume form on $\mathbb{R}^{3}$ ), we have

$$
\varphi=f^{-3} \boldsymbol{b} \boldsymbol{b} \boldsymbol{b}+f \boldsymbol{b} \boldsymbol{c} \text { and } * \varphi=\boldsymbol{b} \boldsymbol{c} \boldsymbol{c}+f^{4} \boldsymbol{c c} .
$$

As before, smoothness of $\varphi$ and $* \varphi$ follows from the fact that the coefficient functions are smooth even functions of the distance from the zero section.

More generally, we may ask when an SO(5)-invariant 3- or 4-form on $\mathbb{V} \backslash S^{4}$ extends smoothly to the zero section. By [7, Thm. 4.6] the invariant 3- and 4-forms can be expressed as

$$
p_{1} \boldsymbol{b} \boldsymbol{c}+p_{2} \boldsymbol{b} \boldsymbol{b} \boldsymbol{b}+p_{3} \boldsymbol{a} \boldsymbol{b} \boldsymbol{c}+p_{4} \boldsymbol{a} \boldsymbol{b} \boldsymbol{a c}
$$

and

$$
q_{1} c c+q_{2} b b c+q_{3} a b b c+q_{4} a b a b c,
$$

respectively. Smoothness then amounts to the functions $p_{i}$ and $q_{i}$ being smooth even functions of $r$; again we are using that the above basis elements are adapted to the filtration of $\mathrm{SO}(4)$-equivariant homogeneous polynomials $V \supset S^{2} \rightarrow \Lambda^{p}(T \oplus V), p=3,4$.

Remark 3.6. In addition to the above, [4] provides a similar construction of a complete $\mathrm{G}_{2}$-holonomy metric on $\Lambda_{-}^{2} \mathbb{C P}(2)$; whilst for $S^{4}$ the construction works with both bundles $\Lambda_{ \pm}^{2} S^{4}$, this is not the case for $\mathbb{C P}(2)$ in the sense that one needs to take $\overline{\mathrm{CP}(2)}$ to use $\Lambda_{+}^{2}$.

Let us consider the $\mathrm{SO}(3)_{-}$-bundle over $S^{4}, \mathrm{SO}(5) / \mathrm{SO}(3)_{+} \rightarrow S^{4}$; the following $\mathrm{G}_{2}$-instanton on this space was also considered in [24]. If we regard the canonical connection of this bundle as an $\mathfrak{s o}(3)_{-}$-valued 1 form on $\mathrm{SO}(5) \times \mathrm{SO}_{(3)_{+}} V$, given by

$$
A(t)=\left(\begin{array}{ccc}
0 & -\gamma^{1} & -\gamma^{2} \\
\gamma^{1} & 0 & \gamma^{3} \\
\gamma^{2} & -\gamma^{3} & 0,
\end{array}\right)
$$

it is $\mathrm{SO}(4)$-invariant and has $A^{\prime}(t)=0$. Then, using $d \gamma^{1}=\gamma^{23}-\left(v^{12}-\right.$ $\left.v^{34}\right)$ etc., we find that

$$
F_{A}(t)=\left(\begin{array}{ccc}
0 & v^{12}-v^{34} & v^{13}-v^{42} \\
-v^{12}+v^{34} & 0 & -v^{14}+v^{23} \\
-v^{13}+v^{42} & v^{14}-v^{23} & 0
\end{array}\right) .
$$

Since $F_{A} \wedge \gamma=0$, we see that $A(t)$ is a static solution the instanton evolution equations (indeed, it is the "lift" of an SU(3)-instanton on $\mathbb{C P}(3)$ ). 
In terms of an $r$-dependent frame, we can write the associated curvature 2-form as:

$$
\Omega(r)=f^{-2}\left(\begin{array}{ccc}
0 & -e^{34}+e^{56} & -e^{36}+e^{45} \\
e^{34}-e^{56} & 0 & -e^{35}+e^{46} \\
e^{36}-e^{45} & e^{35}-e^{46} & 0
\end{array}\right)
$$

in particular, we have $\|\Omega(r)\|_{g(r)} \rightarrow 0$ as $r \rightarrow \infty$.

We should mention that [24] includes another (non static) example of a $\mathrm{G}_{2}$-instanton on an $\mathrm{SU}(2)$-bundle over $\Lambda_{+}^{2} S^{4}$; of course this can also be reproduced using our instanton evolution Equation (3.6).

3.5. The spinor bundle over $S^{3}$. We want to construct $\mathrm{G}_{2}$-metrics on cohomogeneity one spaces with principal orbits $S^{3} \times S^{3} \cong \operatorname{Sp}(1)_{+} \times \operatorname{Sp}(1)_{-}$ and singular orbit $S^{3}$, corresponding to stabiliser $\mathrm{Sp}(1)$ with Lie algebra given by the diagonal $\mathfrak{s p}(1) \subset \mathfrak{s p}(1)_{+} \oplus \mathfrak{s p}(1)_{-}$, where

$$
\begin{aligned}
& \mathfrak{s p}(1)=\left\langle\eta_{+}^{1}+\eta_{-}^{1}, \eta_{+}^{2}+\eta_{-}^{2}, \eta_{+}^{3}+\eta_{-}^{3}\right\rangle, \\
& \mathfrak{s p}(1)^{\perp}=\left\langle\eta_{+}^{1}-\eta_{-}^{1}, \eta_{+}^{2}-\eta_{-}^{2}, \eta_{+}^{3}-\eta_{-}^{3}\right\rangle
\end{aligned}
$$

and $d \eta_{ \pm}^{1}=2 \eta_{ \pm}^{23}=2 \eta_{ \pm}^{2} \wedge \eta_{ \pm}^{3}$ etc.

The first known complete $\mathrm{G}_{2}$-metric arising in this context was constructed by Bryant and Salamon in [4]. To obtain their example, we must take $d t=-\sqrt{2} g d r, e^{2 j-1}=f\left(\eta_{+}^{j}-\eta_{-}^{j}\right) / \sqrt{2}$ and $e^{2 j}=r g\left(\eta_{+}^{j}+\eta_{-}^{j}\right) / \sqrt{2}$, for $j=1,2,3$. The corresponding $\mathrm{SU}(3)$-structure is then given by

$$
\begin{gathered}
\sigma=r f g\left(\eta_{+}^{1} \eta_{-}^{1}+\eta_{+}^{2} \eta_{-}^{2}+\eta_{+}^{3} \eta_{-}^{3}\right), \\
\gamma=\frac{f\left(f^{2}-3 r^{2} g^{2}\right)}{2 \sqrt{2}}\left(\eta_{+}^{123}-\eta_{-}^{123}\right) \\
\quad-\frac{f\left(f^{2}+r^{2} g^{2}\right)}{4 \sqrt{2}} d\left(\eta_{+}^{1} \eta_{-}^{1}+\eta_{+}^{2} \eta_{-}^{2}+\eta_{+}^{3} \eta_{-}^{3}\right), \\
\hat{\gamma}=\frac{r g\left(3 f^{2}-r^{2} g^{2}\right)}{2 \sqrt{2}}\left(\eta_{+}^{123}+\eta_{-}^{123}\right)-\frac{r g\left(f^{2}+r^{2} g^{2}\right)}{2 \sqrt{2}}\left(\eta_{+}^{1} \eta_{+}^{2} \eta_{-}^{3}+\eta_{+}^{3} \eta_{+}^{1} \eta_{-}^{2}\right. \\
\left.\quad+\eta_{+}^{1} \eta_{-}^{2} \eta_{-}^{3}+\eta_{+}^{2} \eta_{+}^{3} \eta_{-}^{1}+\eta_{+}^{2} \eta_{-}^{3} \eta_{-}^{1}+\eta_{+}^{3} \eta_{-}^{1} \eta_{-}^{2}\right) .
\end{gathered}
$$

From the above, we see that Equation (3.3) is equivalent to the ODEs

$$
\frac{\partial f}{\partial r}=2 r g^{2} f^{-1}, \quad \frac{\partial g}{\partial r}=-r g^{3} f^{-2},
$$

with corresponding solution:

$$
f(r)=3^{1 / 3}\left(k_{1}^{2} r^{2}+k_{2}\right)^{1 / 3}, \quad g(r)=k_{1} 3^{-1 / 6}\left(k_{1}^{2} r^{2}+k_{2}\right)^{-1 / 6} .
$$

Completeness requires that $k_{2}>0$. For concreteness we shall take $k_{1}=$ $1=k_{2}$ so that $f=g^{-2}$.

In order to understand the geometry near the singular orbit, we consider the vector bundle

$$
\mathbb{V}=\left(\operatorname{Sp}(1)_{+} \times \operatorname{Sp}(1)_{-}\right) \times_{\mathrm{Sp}(1)} V
$$


where the fibres $V \cong \mathbb{H}$ correspond to the standard representation of $\operatorname{Sp}(1)$ and $T=\frac{\mathfrak{s p}(1)_{+} \oplus \mathfrak{s p}(1)_{-}}{\mathfrak{s p}(1)} \cong\left\langle\eta_{+}^{j}-\eta_{-}^{j}\right\rangle$.

As usual, we have the fibre coordinate letter $\boldsymbol{a}$ and its covariant derivative, the letter $\boldsymbol{b}$. Now, choose the map $\Psi: \operatorname{Sp}(1)_{+} \times \mathrm{Sp}(1)_{-} \times \mathbb{R} \rightarrow$ $\mathrm{Sp}(1)_{+} \times \mathrm{Sp}(1)_{-} \times V$ given by $\Psi(g, r)=(g,(r, 0,0,0))$. From this, we find that $\Psi^{*}\left(b_{1}\right)=d r, \Psi^{*}\left(b_{2}\right)=r\left(\eta_{+}^{1}+\eta_{-}^{1}\right) / \sqrt{2}, \Psi^{*}\left(b_{3}\right)=r\left(\eta_{+}^{2}+\eta_{-}^{2}\right) / \sqrt{2}$ and $\Psi^{*}\left(b_{4}\right)=r\left(\eta_{+}^{3}+\eta_{-}^{3}\right) / \sqrt{2}$.

To describe the Bryant-Salamon 3-form in these terms, we need the two elements spanning $\Lambda^{3}(T \oplus V)^{\operatorname{Sp}(1)}=\Lambda^{3}(T) \oplus\left(T \otimes \Lambda^{2} V\right)^{\operatorname{Sp}(1)}=\langle\boldsymbol{v}\rangle \oplus$ $\left\langle\Sigma_{1}\right\rangle$. Similarly, for the 4-form, we need the two non-trivial elements of $\Lambda^{4}(T \oplus V)^{\mathrm{Sp}(1)}=\left(\Lambda^{2}(T) \otimes \Lambda^{2}(V)\right)^{\mathrm{Sp}(1)} \oplus \Lambda^{4}(V)=\left\langle\Sigma_{2}\right\rangle \oplus\langle\boldsymbol{b} \boldsymbol{b} \boldsymbol{b} \boldsymbol{b}\rangle$. Using these forms, defined up to scaling, we can write:

$$
\begin{gathered}
\varphi=(1+\boldsymbol{a} \boldsymbol{a}) \boldsymbol{v}+\Sigma_{1}, \\
* \varphi=(1+\boldsymbol{a} \boldsymbol{a})^{-2 / 3} \boldsymbol{b} \boldsymbol{b} \boldsymbol{b} \boldsymbol{b}+(1+\boldsymbol{a} \boldsymbol{a})^{1 / 3} \Sigma_{2} .
\end{gathered}
$$

The coefficient functions are smooth even functions of the distance from the zero section, again implying that these forms extend smoothly to the zero section of $\mathbb{V}$.

In the above model, the principal stabiliser is trivial. As a consequence, the space of invariant $p$-forms is relatively large, so we shall not write down the general expressions for invariant 3- and 4-forms that extend smoothly to the zero section. It is worthwhile mentioning, however, that the high degree of flexibility leads to (at least) one other complete $\mathrm{G}_{2}$ holonomy metric [2]; this metric has a different behaviour far away from the zero section: it is similar to the Taub-NUT metric in the sense that it is asymptotically locally conical.

We now address the construction of instantons on an $\operatorname{Sp}(1)$-bundle over the Bryant-Salamon $\mathrm{G}_{2}$-structure on the spinor bundle over $S^{3}$. Instantons on this space were also the subject of [6]. The construction is reminiscent of that of the Eguchi-Hanson space in Section 2.4. Motivated by the expression for the canonical connection on the natural $\mathrm{Sp}(1)$ bundle $\mathrm{Sp}(1)_{+} \times \mathrm{Sp}(1)_{-} \rightarrow S^{3}$ over the singular orbit, we consider the one-parameter family of connections specified by the potential

$$
A(t)=p(t)\left(i e^{2}(t)+j e^{4}(t)+k e^{6}(t)\right),
$$

corresponding to a connection on the bundle $\mathrm{Sp}(1)_{+} \times \mathrm{Sp}(1)_{-} \times V \rightarrow \mathbb{V}$.

Straightforward computations give that

$$
\begin{aligned}
A^{\prime}(t) & =-\frac{1}{\sqrt{2} g}\left(\frac{\partial p}{\partial r}+p r^{-1} g^{-1} \frac{\partial(r g)}{\partial r}\right)\left(i e^{2}+j e^{4}+k e^{6}\right), \\
F_{A} & =i \sqrt{2} p\left(r g^{5} e^{35}+\left(\sqrt{2} p+r^{-1} g^{-1}\right) e^{46}\right) \\
& +j \sqrt{2} p\left(r g^{5} e^{51}+\left(\sqrt{2} p+r^{-1} g^{-1}\right) e^{62}\right) \\
& +k \sqrt{2} p\left(r g^{5} e^{13}+\left(\sqrt{2} p+r^{-1} g^{-1}\right) e^{24}\right), \\
*_{t}\left(F_{A} \wedge \gamma\right) & =-\sqrt{2} p\left(\sqrt{2} p+r^{-1} g^{-1}-r g^{5}\right)\left(i e^{2}+j e^{4}+k e^{6}\right) .
\end{aligned}
$$


The instanton evolution equation is then the following non-linear ODE of Bernoulli type:

$$
\frac{\partial p}{\partial r}=2 \sqrt{2} g p^{2}+\left(2 r^{-1}-2 r g^{6}-r^{-1} g^{-1} \frac{\partial(r g)}{\partial r}\right) p .
$$

We find the following non-zero solution

$$
p(r)=\frac{2 r}{2 c\left(1+r^{2}\right)^{1 / 6}-3^{5 / 6} \sqrt{2}\left(1+r^{2}\right)^{5 / 6}},
$$

which is a smooth function of $r \geqslant 0$, so long as we choose our constant $c<3^{5 / 6} / \sqrt{2}$. Clearly $\lim _{r \rightarrow 0} A(r)=0$.

For the curvature, we find that

$$
\Omega(r)=2 p\left(\sqrt{2} g p+r^{-1}-r g^{6}\right)\left(i d r \wedge e^{2}+j d r \wedge e^{4}+k d r \wedge e^{6}\right)+F_{A}(r)
$$

which vanishes at the zero section and satisfies $\|\Omega(r)\|_{g(r)} \rightarrow 0$ as $r \rightarrow \infty$.

Slightly more generally, as in [6], we could consider the potential

$$
A(t)=p(t)\left(i e^{1}(t)+j e^{3}(t)+k e^{5}(t)\right)+q(t)\left(i e^{2}(t)+j e^{4}(t)+k e^{6}(t)\right) .
$$

As in op. cit., we find that solutions that extend smoothly to the zero section necessarily have $q \equiv 0$.

Computations for the Brandhuber et. al. $\mathrm{G}_{2}$-metric on $\mathbb{V} \cong S^{3} \times \mathbb{H}$ [2] show that there is a globally defined instanton on a circle bundle over this space.

\section{THE $\operatorname{Spin}(7)$ CASE}

4.1. Spin(7)-structures. From a family of $\mathrm{G}_{2}$-structures $I \ni \rightarrow \varphi(t)$ on a 7 -manifold $N$, we can construct a $\operatorname{Spin}(7)$-structure on the product $M=$ $I \times N$ via

$$
\Phi=\varphi \wedge d t+* \varphi .
$$

In terms of Equation (4.1), the condition (1.6) for the induced metric to have holonomy contained in $\operatorname{Spin}(7)$ amounts to requiring that $d *_{t} \varphi(t)=$ 0 for each $t$ (i.e. that $\varphi(t)$ is co-calibrated) and that the family satisfies the evolution equations [20]:

$$
\frac{\partial(* \varphi)}{\partial t}=-d \varphi
$$

This preserves closedness of $* \varphi$ and therefore gives us a way of evolving an initial co-calibrated $\mathrm{G}_{2}$-structure on $N$ to give our torsion-free $\operatorname{Spin}(7)$ structure. This is again sometimes referred to as "Hitchin's flow equation".

4.2. Spin(7)-instantons. Given a Spin(7)-manifold $M=I \times N$ of the form (4.1), we can rephrase the instanton condition for a connection $\omega$ on the pullback of a bundle $Q$ on $N$ as follows:

Proposition 4.1. In terms of t-dependent data on $N$, the Spin(7)-instanton condition (1.7) is given by:

$$
A^{\prime}=*_{t}\left(F_{A} \wedge *_{t} \varphi\right)
$$


Proof. By Equation (1.7), we have to compute $\Phi \wedge \Omega=-* \Omega$ in terms of the $t$-dependent data. We get two equations:

$$
A^{\prime}=*_{t}\left(F_{A} \wedge *_{t} \varphi\right) \text { and } *_{t} \varphi \wedge A^{\prime}=*_{t} F_{A}+\varphi \wedge F_{A} .
$$

If $V$ denotes the irreducible 7-dimensional representation of $\mathrm{G}_{2}$ then the following two equivariant maps $\Lambda^{2} V \rightarrow \Lambda^{5} V$ coincide:

$$
\beta \mapsto * \varphi \wedge *(\beta \wedge * \varphi) \text { and } \beta \mapsto * \beta+\beta \wedge \varphi \text {. }
$$

The assertion now follows.

As Equation (4.3) is in Cauchy form, we have:

Corollary 4.2. Given real analytic initial data, Equation (4.3) has a unique solution over an open subset of $I \times N$.

From the form of Equation (4.3), we see from Equation (1.5) that:

Corollary 4.3. If $\omega$ is asymptotic to a connection on $Q \rightarrow N$ at an endpoint of $I$, then the limiting connection is a $\mathrm{G}_{2}$-instanton.

4.3. Flat $\mathbb{R}^{8}$. To obtain the standard $\operatorname{Spin}(7)$-structure on $\mathbb{R}^{8}$ defining the flat metric, we will evolve the standard $\mathrm{G}_{2}$-structure on $S^{7}$ which satisfies $d \varphi=-4 * \varphi$ (a so-called "nearly parallel $\mathrm{G}_{2}$-structure"). Equation (4.2) quickly yields that the evolving $\mathrm{G}_{2}$-structures are $\varphi(t)=t^{3} \varphi$ so that the Spin(7)-structure is

$$
\Phi=t^{3} \varphi \wedge d t+t^{4} * \varphi
$$

for $t>0$. This is a conical solution which always occurs when the initial 7dimensional hypersurface is endowed with a nearly parallel $\mathrm{G}_{2}$-structure. Based on this description of $\mathbb{R}^{8}$ and a suitable ansatz for $A$, we can use Equation (4.3) so as to obtain the "basic" Spin(7)-instanton on the trivial bundle $\operatorname{Spin}(7) \times \mathbb{R}^{8}$ that appeared in $[12,15,22]$.

We could also view $\mathbb{R}^{8}=\mathbb{R}^{4} \times \mathbb{R}^{4}$ and take as hypersurfaces $S^{3} \times \mathbb{R}^{4}$. If we let $\eta^{1}, \eta^{2}, \eta^{3}$ be the coframe on $S^{3}$ with $d \eta^{1}=-2 \eta^{23}$ etc. and let $\tau_{1}, \tau_{2}, \tau_{3}$ be the standard hyperKähler triple on $\mathbb{R}^{4}$, we may take the ansatz

$$
\varphi(t)=f(t)^{3} \eta^{1} \wedge \eta^{2} \wedge \eta^{3}+f(t)\left(\tau_{1} \wedge \eta^{1}+\tau_{2} \wedge \eta^{2}+\tau_{3} \wedge \eta^{3}\right)
$$

for our $\mathrm{G}_{2}$-structures on $S^{3} \times \mathbb{R}^{4}$. Equation (4.2) yields $f(t)=-t$ as a solution, which is equivalent to choosing the evolving coframe $e(t)=-t \eta$ on $S^{3}$ as expected. Hence, if we write $\sigma_{1}=t d t \wedge \eta^{1}-t^{2} \eta^{2} \wedge \eta^{3}$ etc., so that $\left(\sigma_{1}, \sigma_{2}, \sigma_{3}\right)$ is the standard hyperKähler triple on $\mathbb{R}^{4}$, then

$$
\Phi=\frac{1}{6}\left(\sigma_{1}^{2}+\sigma_{2}^{2}+\sigma_{3}^{2}\right)+\sigma_{1} \wedge \tau_{1}+\sigma_{2} \wedge \tau_{2}+\sigma_{3} \wedge \tau_{3}-\frac{1}{6}\left(\tau_{1}^{2}+\tau_{2}^{2}+\tau_{3}^{2}\right)
$$

is the solution, which is a re-writing of the standard Spin(7)-form. If we choose our evolving connections $A(t)=-a(t) t\left(i \eta^{1}+j \eta^{2}+k \eta^{3}\right)$ on $S^{3} \times \mathbb{R}^{4}$, the Spin(7)-instanton evolution equation (4.3) will yield the pullback of the basic (SD) instanton on $\mathbb{R}^{4}$, as expected from Remarks 1.2 and 1.3. This situation is equivalent to considering $\mathbb{R}^{8}$ as the (negative) spinor bundle of $\mathbb{R}^{4}$ and taking the sphere subbundles as hypersurfaces. We will see a closely related construction in Section 4.4 which yields nontrivial results. 
4.4. The spinor bundle over $S^{4}$. Let us consider the 7-sphere, eventually leading to the Bryant-Salamon metric on the negative spinor bundle over $S^{4}$ [4]. Similarly to Equation (3.7) we write $S^{7}=S p(2) / S p(1)+$ using the explicit identification

$$
\left(\begin{array}{cccccccc}
0 & -\gamma^{1} & -\gamma^{2} & -\gamma^{3} & -v^{1} & -v^{2} & -v^{3} & -v^{4} \\
\gamma^{1} & 0 & -\gamma^{3} & \gamma^{2} & v^{2} & -v^{1} & -v^{4} & v^{3} \\
\gamma^{2} & \gamma^{3} & 0 & -\gamma^{1} & v^{3} & v^{4} & -v^{1} & -v^{2} \\
\gamma^{3} & -\gamma^{2} & \gamma^{1} & 0 & v^{4} & -v^{3} & v^{2} & -v^{1} \\
v^{1} & -v^{2} & -v^{3} & -v^{4} & 0 & -\eta^{1} & -\eta^{2} & -\eta^{3} \\
v^{2} & v^{1} & -v^{4} & v^{3} & \eta^{1} & 0 & -\eta^{3} & \eta^{2} \\
v^{3} & v^{4} & v^{1} & -v^{2} & \eta^{2} & \eta^{3} & 0 & -\eta^{1} \\
v^{4} & -v^{3} & v^{2} & v^{1} & \eta^{3} & -\eta^{2} & \eta^{1} & 0
\end{array}\right),
$$

where $\mathfrak{s p}(1)_{+}=\left\langle\eta^{1}, \eta^{2}, \eta^{3}\right\rangle$. The associated structure equations imply that

$$
\begin{gathered}
d \gamma^{1}=2\left(-\gamma^{23}+v^{12}-v^{34}\right), \quad d \gamma^{2}=2\left(-\gamma^{31}+v^{13}-v^{42}\right), \\
d \gamma^{3}=2\left(-\gamma^{12}+v^{14}-v^{23}\right), \\
d\left(v^{12}-v^{34}\right)=-2 \gamma^{2}\left(v^{14}-v^{23}\right)+2 \gamma^{3}\left(v^{13}-v^{42}\right), \\
d\left(v^{13}-v^{42}\right)=2 \gamma^{1}\left(v^{14}-v^{23}\right)-2 \gamma^{3}\left(v^{12}-v^{34}\right), \\
d\left(v^{14}-v^{23}\right)=-2 \gamma^{1}\left(v^{13}-v^{42}\right)+2 \gamma^{2}\left(v^{12}-v^{34}\right),
\end{gathered}
$$

and then

$$
\begin{gathered}
d \gamma^{123}=2 \gamma^{23}\left(v^{12}-v^{34}\right)+2 \gamma^{31}\left(v^{13}+v^{24}\right)+2 \gamma^{12}\left(v^{14}-v^{23}\right) \\
d\left(\gamma^{1}\left(v^{12}-v^{34}\right)+\gamma^{2}\left(v^{13}+v^{24}\right)+\gamma^{3}\left(v^{14}-v^{23}\right)\right)=-12 v^{1234} \\
\quad+2 \gamma^{23}\left(v^{12}-v^{34}\right)+2 \gamma^{12}\left(v^{14}-v^{23}\right)+2 \gamma^{31}\left(v^{13}-e^{42}\right) .
\end{gathered}
$$

We now look for an ansatz with $d t=f^{1 / 2} d r, e^{j}=r f^{1 / 2} \gamma^{j}, j=1,2,3$, and $e^{4}=g^{1 / 2} v^{1}, e^{5}=g^{1 / 2} v^{2}, e^{6}=g^{1 / 2} e^{3}, e^{7}=g^{1 / 2} v^{4}$ so that the associated $\mathrm{G}_{2}$-structure reads

$$
\begin{aligned}
& \varphi=r^{3} f^{3 / 2} \gamma^{123}+r f^{1 / 2} g\left(\gamma^{1}\left(v^{12}-v^{34}\right)+\gamma^{2}\left(v^{13}-v^{42}\right)+\gamma^{3}\left(v^{14}-v^{23}\right)\right), \\
& * \varphi=g^{2} v^{1234}-r^{2} f g\left(\gamma^{23}\left(v^{12}-v^{34}\right)+\gamma^{31}\left(v^{13}-v^{42}\right)+\gamma^{12}\left(v^{14}-v^{23}\right)\right) .
\end{aligned}
$$

Using the above computations, we can verify that $\varphi$ is co-calibrated, i.e. $d * \varphi=$ 0 . The evolution equations (4.2) are

$$
\frac{\partial g}{\partial r}=6 r f, \quad \frac{\partial f}{\partial r}=-4 r f^{2} g^{-1}
$$

which then have the solution

$$
f(r)=\left(\frac{2}{5}\right)^{2 / 5}\left(\frac{2 r^{2}-k_{1} k_{2}}{k_{1}}\right)^{-2 / 5}, g(r)=\left(\frac{5}{2}\right)^{3 / 5} k_{1}\left(\frac{2 r^{2}-k_{1} k_{2}}{k_{1}}\right)^{3 / 5} .
$$

For concreteness, let us fix $k_{1}=1$ and $k_{2}=-2$, giving

$$
f(r)=5^{-2 / 5}\left(1+r^{2}\right)^{-2 / 5}, \quad g(r)=5^{3 / 5}\left(1+r^{2}\right)^{3 / 5} .
$$

Note that for this choice of integration constants, we have $f(r)^{3}=g(r)^{-2}$, and this will lead to a complete holonomy Spin(7)-metric. 
Turning to the geometry near the singular orbit, we need to study the vector bundle

$$
\mathbb{V}=\mathrm{Sp}(2) \times \mathrm{Sp}(1)_{+} \times \mathrm{Sp}(1)_{-} V
$$

where the fibres $V=\mathbb{R}^{4} \cong \mathbb{H}$ are the standard representation of $\operatorname{Sp}(1)$ (again acting on the right); in our conventions $\mathfrak{s p}(1)_{-}=\left\langle\gamma^{1}, \gamma^{2}, \gamma^{3}\right\rangle$. Obviously, the volume on $T \cong\left\langle v^{1}, v^{2}, v^{3}, v^{4}\right\rangle$ gives us an invariant 4-form $v$ on $\mathbb{V}$, and relevant letters of our dictionary are the fibre coordinates $\boldsymbol{a}$ and its covariant derivative $\boldsymbol{b}$.

Using contraction via the volume form on $\mathbb{R}^{4}$, we get the invariant 4form $\boldsymbol{b} \boldsymbol{b} \boldsymbol{b} \boldsymbol{b}$. In addition, note that we have a map $\Sigma_{1}: V \otimes V \rightarrow \Sigma_{-}^{2}$ and a similar map $\widetilde{\Sigma}_{1}: T \otimes T \rightarrow \Sigma_{-}^{2}$. This means there is an invariant 4 -form $\Sigma_{1}(\boldsymbol{b}, \boldsymbol{b}) \widetilde{\Sigma}_{1}(\boldsymbol{v}, \boldsymbol{v})$ corresponding to the contraction $\Sigma_{-}^{2} \otimes \Sigma_{-}^{2} \rightarrow \mathbb{R}$.

As in the previous cases, we use a map $\Psi: \operatorname{Sp}(2) \times \mathbb{R} \rightarrow \mathrm{Sp}(2) \times V$, now given by $\Psi(g, r)=(g,(r, 0,0,0))$, so as to get $\Psi^{*}\left(b_{0}\right)=d r, \Psi^{*}\left(b_{1}\right)=r \gamma^{1}$, $\Psi^{*}\left(b_{2}\right)=r \gamma^{3}$ and $\Psi^{*}\left(b_{3}\right)=r \gamma^{2}$. In invariant terms, we can then express the 4 -form $\Phi$ by

$$
\begin{gathered}
\Phi=(1+\boldsymbol{a} \boldsymbol{a})^{-4 / 5} \boldsymbol{b} \boldsymbol{b} \boldsymbol{b} \boldsymbol{b} \\
+(1+\boldsymbol{a} \boldsymbol{a})^{1 / 5} \Sigma_{1}(\boldsymbol{b}, \boldsymbol{b}) \widetilde{\Sigma}_{1}(\boldsymbol{v}, \boldsymbol{v})+(1+\boldsymbol{a} \boldsymbol{a})^{6 / 5} \boldsymbol{v},
\end{gathered}
$$

where, as usual, we have chosen suitable rescalings of the invariant forms. As the coefficient functions are smooth even functions of the distance from the zero section, this form extends to smoothly to the zero section, by the usual arguments.

Let us now turn to the construction of instantons on $\mathrm{Sp}(1)$-bundles over $\mathbb{V}$. Examples of such instantons were also discussed in $[6,23]$. On the bundle $\mathrm{Sp}(2) \times{ }_{\mathrm{Sp}(1)_{+}} V \rightarrow \mathbb{V}$, we can consider a connection corresponding to the following one-parameter family of potentials (defined along the principal orbits):

We then have

$$
A(t)=p(t)\left(i e^{1}+j e^{2}+k e^{3}\right) .
$$

$$
\begin{aligned}
A^{\prime}(t) & =\left(f^{-1 / 2} \frac{\partial p}{\partial r}+\frac{p}{r f} \frac{\partial\left(r f^{1 / 2}\right)}{\partial r}\right)\left(i e^{1}+j e^{2}+k e^{3}\right), \\
F_{A}= & 2 i p\left(\left(p-r^{-1} f^{-1 / 2}\right) e^{23}+r f^{2}\left(e^{45}-e^{67}\right)\right) \\
& +2 j p\left(\left(p-r^{-1} f^{-1 / 2}\right) e^{31}+r f^{2}\left(e^{46}-e^{75}\right)\right) \\
& +2 k p\left(\left(p-r^{-1} f^{-1 / 2}\right) e^{12}+r f^{2}\left(e^{47}-e^{56}\right)\right) .
\end{aligned}
$$

Straightforward computations show that

$$
*_{t}\left(F_{A} \wedge *_{t} \varphi\right)=2 p\left(p-r^{-1} f^{-1 / 2}+2 r f^{2}\right)\left(i e^{1}+j e^{2}+k e^{3}\right) .
$$

So the instanton evolution equation reads:

$$
\frac{\partial p}{\partial r}=2 f^{1 / 2} p^{2}-p\left(2 r^{-1}-4 r f^{5 / 2}+\frac{1}{r f^{1 / 2}} \frac{\partial\left(r f^{1 / 2}\right)}{\partial r}\right)
$$

which is a Bernoulli equation. 
It is possible to solve explicitly for $p$; the solution can be expressed in terms of the generalised hypergeometric function $x \mapsto{ }_{2} F_{1}\left(1,1 ; \frac{8}{5} ; x\right)$. Explicitly, we have that $p(r)$ is given by

$$
\frac{15\left(1+r^{2}\right)^{6 / 5}}{3 r\left(r^{2}\left(5 c\left(1+r^{2}\right)^{3 / 5}+5^{4 / 5}\right)+5^{4 / 5}\right)+25^{4 / 5} r\left(1+r^{2}\right){ }_{2} F_{1}\left(1,1 ; \frac{8}{5} ;-r^{-2}\right)} \text {. }
$$

For suitably chosen $c \in \mathbb{R}$, the limiting behaviour of this solution is, in a sense, very similar to that of our $\mathrm{SO}(3)$-invariant instanton on the Eguchi-Hanson space. In particular, we find that

$$
\lim _{r \rightarrow 0} A(r)=i \gamma^{1}+j \gamma^{2}+k \gamma^{3}
$$

corresponding to the canonical connection of the $\operatorname{Sp}(1)$-bundle $\operatorname{Sp}(2) /$ $\operatorname{Sp}(1)_{+} \rightarrow S^{4}$. Far away from the zero section, we find that $\|\Omega(r)\|_{g(r)} \rightarrow 0$ as $r \rightarrow \infty$.

There also an instanton on the bundle $\mathrm{Sp}(2) \times \mathrm{Sp}(1)_{-} \mathbb{H} \rightarrow \mathbb{V}$. To see this, consider the connection form

$$
A(t)=\left(\begin{array}{ccc}
0 & -\eta^{1} & -\eta^{2} \\
\eta^{1} & 0 & -\eta^{3} \\
\eta^{2} & \eta^{3} & 0
\end{array}\right)
$$

which has $A^{\prime}(t)=0$. Straightforward computations show that $F_{A} \wedge *_{t} \varphi=$ 0 , giving that $A$ solves the instanton evolution equations statically.

To find other explicit examples of Spin(7)-instantons, one could consider other known complete Spin(7)-metrics, obtainable via Equation (4.2). Some of these arise in the context of cohomogeneity one SU(3)-actions (see, for instance, $[18,25])$. Other examples, including an analogue of the Taub-NUT metric, were studied in [10]. As the associated metrics have a more complicated asymptotic behaviour (asymptotically locally conical), we do not expect to get instantons from an ansatz as above. Instead, one should use an approach similar to the one mentioned for the Brandhuber et. al. holonomy $\mathrm{G}_{2}$-metric [2] in Section 3.5.

\section{REFERENCES}

[1] M. Atiyah and N. Hitchin. The geometry and dynamics of magnetic monopoles. M. B. Porter Lectures. Princeton University Press, Princeton, NJ, 1988.

[2] A. Brandhuber, J. Gomis, S.S. Gubser, and S. Gukov. Gauge theory at large $N$ and new $\mathrm{G}_{2}$ holonomy metrics. Nucl. Phys., B, 611(1-3):179-204, 2001.

[3] R. L. Bryant. Non-embedding and non-extension results in special holonomy. In The many facets of geometry, pages 346-367. Oxford Univ. Press, Oxford, 2010.

[4] R. L. Bryant and S. M. Salamon. On the construction of some complete metrics with exceptional holonomy. Duke Math. J., 58(3):829-850, 1989.

[5] M. Mamone Capria and S. M. Salamon. Yang-Mills fields on quaternionic spaces. Nonlinearity, 1(4):517-530, 1988.

[6] A. Clarke. Instantons on the exceptional holonomy manifolds of Bryant and Salamon. J. Geom. Phys., 82:84-97, 2014.

[7] D. Conti. Special holonomy and hypersurfaces. PhD thesis, Scuola Normale Superiore, Pisa, 2005.

[8] D. Conti. Invariant forms, associated bundles and Calabi-Yau metrics. J. Geom. Phys., 57(12):2483-2508, 2007. 
[9] D. Conti and T. B. Madsen. Harmonic structures and intrinsic torsion. Transform. Groups, 20(3):699-723, 2015.

[10] M. Cvetič, G. W. Gibbons, H. Lü, and C. N. Pope. New complete noncompact Spin(7) manifolds. Nucl. Phys., B, 620(1-2):29-54, 2002.

[11] J.-H. Eschenburg and McKenzie Y. Wang. The initial value problem for cohomogeneity one Einstein metrics. J. Geom. Anal., 10(1):109-137, 2000.

[12] D. B. Fairlie and J. Nuyts. Spherically symmetric solutions of gauge theories in eight dimensions. J. Phys. A, 17(14):2867-2872, 1984.

[13] M. Fernandez and A. Gray. Riemannian manifolds with structure group $\mathrm{G}_{2}$. Ann. Mat. Pura Appl. (4), 132:19-45, 1982.

[14] M. Fernández. A classification of Riemannian manifolds with structure group Spin(7). Ann. Mat. Pura Appl. (4), 143:101-122, 1986.

[15] S. Fubini and H. Nicolai. The octonionic instanton. Phys. Lett. B, 155(5-6):369-372, 1985.

[16] A. Gambioli. SU(3)-manifolds of cohomogeneity one. Ann. Global Anal. Geom., 34(1):77-100, 2008.

[17] G. W. Gibbons and P. J. Ruback. The hidden symmetries of multicentre metrics. Comm. Math. Phys., 115(2):267-300, 1988.

[18] S. Gukov and J. Sparks. M-theory on Spin(7) manifolds. Nucl. Phys., B, 625(1-2):3-69, 2002.

[19] M. Günaydin and H. Nicolai. Seven-dimensional octonionic Yang-Mills instanton and its extension to an heterotic string soliton. Phys. Lett. B, 351(1-3):169-172, 1995.

[20] N. J. Hitchin. Stable forms and special metrics. In Global differential geometry: the mathematical legacy of Alfred Gray (Bilbao, 2000), volume 288 of Contemp. Math., pages 70-89. Amer. Math. Soc., Providence, RI, 2001.

[21] N. J. Hitchin. The self-duality equations on a Riemann surface. Proc. Lond. Math. Soc. (3), 55:59-126, 1987.

[22] T. A. Ivanova and A. D. Popov, (Anti)self-dual gauge fields in dimension $d \geqslant 4$, Teoret. Mat. Fiz., 94(2):316-342, 1993.

[23] H. Kanno and Y. Yasui. Octonionic Yang-Mills instanton on quaternionic line bundle of Spin(7) holonomy. J. Geom. Phys., 34(3-4):302-320, 2000.

[24] G. Oliveira. Monopoles on the Bryant-Salamon $\mathrm{G}_{2}$-manifolds. J. Geom. Phys., 86:599632, 2014.

[25] F. Reidegeld. Exceptional holonomy and Einstein metrics constructed from AloffWallach spaces. Proc. Lond. Math. Soc. (3), 102(6):1127-1160, 2011.

[26] R. Reyes Carrión. A generalization of the notion of instanton. Differential Geom. Appl., 8(1):1-20, 1998.

(J.D. Lotay) Department of Mathematics, University College London, Gower Street, London WC1E 6BT, United Kingdom.

E-mail address: j.lotay@ucl.ac.uk

(T. B. Madsen) Department of Mathematics, Aarhus University, Ny Munkegade 118, Bldg 1530, 8000 Aarhus, Denmark.

E-mail address: thomas.madsen@math.au.dk 